\title{
Uma Abordagem de Estimativa de Software Baseada em Produtividade por Categoria de Caso de Uso
}

\author{
Paula Franklin Chaves de Sousa ${ }^{2}$, Fabio Pinheiro Abreu ${ }^{1,2}$ \\ ${ }^{1}$ Universidade de Fortaleza - UNIFOR \\ Mestrado em Informática Aplicada - MIA \\ Av. Washington Soares, 1321 \\ CEP 60.811-341, Fortaleza-CE-Brasil \\ ${ }^{2}$ E-NOVAR Soluções Tecnológicas Ltda \\ Rua Costa Barros, 915, $9^{\circ}$. Andar \\ CEP 60.160-280, Fortaleza-CE-Brasil \\ fabiopinheiroabreu@gmail.com, paula@enovar.com.br
}

\begin{abstract}
Resumo. A estimativa de software é um dos maiores desafios para contratantes e fornecedores de TI. A técnica de ponto de caso de uso (PCU) é uma técnica bastante utilizada na estimativa de softwares cujo desenvolvimento se baseia em casos de uso e orientação a objetos. Nessa técnica, a estimativa de tamanho é realizada obtendose a ponderação de atores, casos de uso, fatores técnicos e fatores ambientais. A estimativa de esforço é obtida a partir da multiplicação do tamanho do projeto pela produtividade média da organização. Entretanto, casos de uso podem ter complexidades semelhantes, mas requerem diferentes esforços para desenvolvimento. Este artigo apresenta uma abordagem para estimativa de software baseada em produtividades por categorias de casos de uso e os beneficios dessa abordagem na melhoria do processo de estimativa de software.
\end{abstract}

\begin{abstract}
Software estimation is the major challenge for contractors and vendors. The use case point (UCP) is a widely used technique for estimating software whose development is based on use cases and object orientation. In this technique, the estimated size is accomplished by obtaining the weight of actors, use cases, technical factors and environmental factors. The effort estimate is obtained by multiplying the size of the project by the average productivity of the organization. However, use cases may have similar complexities, but require different efforts for development. This paper presents an approach to estimate software based on productivities for use case categories and benefits of this approach in improving the process of software estimation.
\end{abstract}

\section{$1 \quad$ Introdução}

A estimativa de software é um dos maiores desafios para contratantes e fornecedores de TI. A estimativa é a essência da dificuldade que temos em controlar projetos de software (DEMARCO, 1991). É uma das atividades que apresentam mais incertezas e que exerce grande influência no processo de desenvolvimento de software, pois é através dela que se pode ter a quantidade de pessoas necessárias, o tempo necessário ou o custo previsto do projeto. 
O planejamento e controle de um projeto de software não seriam possíveis sem a realização de estimativas confiáveis, pois não é possível elaborar cronograma e orçamento sem o uso destas. O processo de estimativa de um projeto de software envolve quatro atividades básicas: estimar o tamanho do produto a ser desenvolvido; estimar o esforço empregado na execução do projeto; estimar o prazo (duração) do projeto; estimar o custo do projeto (VAZQUEZ, 2003). Saber com exatidão tais informações muitas vezes só é possível quando o projeto é finalizado. É diante desta realidade que técnicas de estimativas, tais como linhas de código (LOC), pontos de função (PF) e pontos de casos de uso (PCU) são tão importantes e são utilizadas para se obter um melhor planejamento do projeto.

Este trabalho tem como principal objetivo relatar a experiência da E-NOVAR no uso da técnica de estimativa de ponto de caso de uso, evoluindo sua utilização para considerar diferentes produtividades por categorias de casos de uso.

Este artigo está estruturado em seis seções. A seção 2 apresenta o perfil da empresa. A seção 3 descreve as características da técnica de estimativa de ponto de caso de uso. As características da estimativa baseada em produtividade por categoria de caso de uso estão descritas na seção 4. A seção 5 detalha um estudo de caso, as lições aprendidas e os resultados obtidos. Por fim, a seção 6 apresenta a conclusão do trabalho.

\section{Perfil da E-NOVAR}

A E-NOVAR Soluções Tecnológicas surgiu em 2006, em Fortaleza, com o objetivo de trazer soluções tecnológicas inovadoras para o setor de tecnologia da informação, atuando principalmente como fábrica de soluções sob medida para seus clientes. Nesse ano, a E-NOVAR estabeleceu parcerias com empresas de todo o país, as quais a permitiram participar da execução de projetos de porte nacional e internacional.

Em 2008, a E-NOVAR iniciou o processo de certificação no Programa de Melhoria de Processo do Software Brasileiro - mais conhecido como MPS.Br, conquistando essa certificação para o nível de maturidade G em 2009. Ainda em 2009, a E-NOVAR foi a única empresa cearense beneficiada pelo Programa de Subvenção Econômica da Financiadora de Estudos e Projetos (FINEP) na área 1: Tecnologia da Informação.

Recentemente, em Novembro de 2010, a E-NOVAR obteve a recomendação para a certificação ISO 9001:2008 nos projetos de desenvolvimento de software e nas áreas administrativa e de suporte à infraestrutura de tecnologia da informação e em Dezembro desse mesmo ano realizou uma avaliação conjunta nos modelos MPS.Br nível de maturidade E e CMMI - nível de maturidade 2 (com nível de capacidade 3) dos processos de software, obtendo sua oficialização em Janeiro de 2011.

\section{$3 \quad$ Ponto de Caso de Uso}

Ponto de caso de uso (PCU) é uma técnica de estimativa de software cujo desenvolvimento se baseia em casos de uso e orientação a objetos e foi criada por Gustav Karner em 1993 (RIBU K, 2001). Ela se baseia no método de Pontos de Função e em uma adaptação da técnica FPA denominada Mk II FPA. 
A técnica PCU estima o tamanho de um software e é obtida através da ponderação de atores, casos de uso, fatores técnicos e fatores ambientais. $\mathrm{O}$ resultado dessa ponderação é um valor que consiste no tamanho do projeto em PCUs. A estimativa de esforço é obtida a partir da multiplicação do tamanho do projeto pela produtividade da organização (o esforço necessário para desenvolver 1 PCU).

Essa técnica considera que, uma vez que os casos de uso principais do sistema sejam identificados, é possível estimar o tamanho do software como um todo se baseando em um conjunto simples de métricas e modificadores, similar à técnica de Pontos de Função. As formas de manipular estes fatores para se chegar a uma estimativa são descritas a seguir:

\subsection{Ponderação dos Atores}

O primeiro passo da técnica consiste na ponderação dos atores envolvidos no projeto para se obter o peso não ajustado dos atores (UAW). A ponderação dos atores utiliza os seguintes pesos:

Tabela 1 - Ponderação dos Atores (UAW)

\begin{tabular}{|c|l|c|}
\hline Complexidade & \multicolumn{1}{|c|}{ Descrição } & Fator \\
\hline Simples & Disparam programas simples de interface com outros sistemas. & $\mathbf{1}$ \\
\hline Médio & $\begin{array}{l}\text { Programas de interface com outros sistemas, envolvendo algum protocolo para a troca } \\
\text { de informações ou pessoas interagindo com interface não gráfica. }\end{array}$ & $\mathbf{2}$ \\
\hline Complexo & Pessoa interagindo com interface gráfica. & $\mathbf{3}$ \\
\hline
\end{tabular}

O peso total dos atores do sistema é calculado pela soma dos produtos dos atores de cada tipo pelo respectivo peso: $\mathbb{Z}$ AW (Unadjusted Actor Weight) $=\Sigma$ Ponderação total dos Atores.

\subsection{Ponderação dos Casos de Uso}

A próxima ponderação a ser realizada é de caso de uso, onde esta é definida de acordo com o número de transações de acordo com a tabela a seguir:

Tabela 2 - Ponderação dos Casos de Uso (UUCW)

\begin{tabular}{|c|l|c|}
\hline Complexidade & \multicolumn{1}{|c|}{ Descrição } & Fator \\
\hline Simples & 3 ou menos transações & 5 \\
\hline Médio & 4 a 7 transações & 10 \\
\hline Complexo & 8 a 11 transações & 15 \\
\hline N-Complexo & Acima de 11 transações & T = número de transações; $R$ = resto da divisão de $T / 11 ;$ \\
\hline
\end{tabular}

O peso total dos casos de uso é calculado pela soma dos fatores atribuídos a cada caso de uso do sistema: $\mathbb{Z U U C W}$ (Unadjusted Use Case Weight) $=\Sigma$ Fator dos Casos de Uso.

\subsection{Pontos de Caso de Uso Não Ajustados}

Com as duas ponderações calculadas até então é possível obter total de pontos de caso de uso não ajustados (UUCP): UUCP $=\mathbb{Z U U C W}+\mathbb{Z} \mathrm{UAW}$.

Esse índice é denominado ponto de caso de uso não ajustado por ainda não considerar a influência dos fatores técnicos e ambientais. 


\subsection{Fatores de Ajustes}

Existem fatores que irão gerar ajustes na estimativa do sistema. Esses ajustes são similares aos adotados pela técnica de Ponto de Função.

$\mathrm{Na}$ técnica PCU esses fatores se dividem entre aqueles que têm características de requisitos funcionais (fatores técnicos - TCF) e os de características de requisitos não funcionais (fatores ambientais - EF).

Tabela 3 - Ponderação dos Fatores Técnicos

\begin{tabular}{|l|c|l|l|}
\hline \multicolumn{1}{|c|}{ Ponderação Fatores Técnicos } & Peso & \multicolumn{1}{|c|}{ Ponderação Fatores Técnicos } & $\begin{array}{c}\text { Pes } \\
\text { o }\end{array}$ \\
\hline T1 - Sistemas distribuídos & 2 & T8 - Portabilidade & 2 \\
\hline T2 - Tempo de resposta/desempenho & 1 & T9 - Facilidade de manutenção & 1 \\
\hline T3 - Eficiência on-line & 1 & T10 - Acessos simultâneos & 1 \\
\hline T4 - Processamento interno complexo & 1 & T11 - Aspectos especiais de segurança & 1 \\
\hline T5 - Código reutilizável & 1 & T12 - Acesso direto para terceiros & 1 \\
\hline T6 - Facilidade de instalação & 0,5 & T13 - Facilidades especiais de treinamento & 1 \\
\hline T7 - Usabilidade & 0,5 & \multicolumn{2}{|l}{}
\end{tabular}

Tabela 4 - Ponderação dos Fatores Ambientais

\begin{tabular}{|l|c|l|c|}
\hline \multicolumn{1}{|c|}{ Ponderação dos Fatores Ambientes } & Peso & \multicolumn{1}{|c|}{ Ponderação dos Fatores Ambientes } & $\begin{array}{c}\text { Pes } \\
\mathbf{0}\end{array}$ \\
\hline E1 - Familiaridade com o processo de desenvolvimento & 1,5 & E5 - Motivação & 1 \\
\hline E2 - Experiência na aplicação & 0,5 & E6 - Estabilidade dos requisitos & 2 \\
\hline E3 - Experiência em OO & 1 & E7 - Membros da equipe com dedicação parcial & -1 \\
\hline E4 - Experiência do líder do projeto & 0,5 & E8 - Dificuldade da linguagem de programação & -1 \\
\hline
\end{tabular}

Em ambos os casos, para cada fator listado é preciso atribuir uma nota entre 0 a 5 (significando: 0 - Não ou irrelevante, 1 - Pouco, 2 - Razoável, 3 - Médio ou influencia moderada, 4 - Muito, 5 - Elevado ou forte influencia) que deve ser utilizada nas fórmulas:

$$
\mathbf{T C F}=0,6+\left(0,01 * \Sigma \text { Peso }^{*} \text { Nota Ti }\right) \text { e } \mathbf{E F}=1,4+\left(-0,03 * \Sigma \text { Peso }^{*} \text { Nota Ei }\right)
$$

\subsection{Calculo dos Pontos de Caso de Uso}

A estimativa de tamanho, representada pelo ponto de caso de uso ajustado (PCU), é obtida através do produto dos pontos de caso de uso não ajustados pelos fatores de ajustes: $\mathrm{PCU}=\mathrm{UUCP}^{*} \mathrm{TCF} * \mathrm{EF}$.

\subsection{Estimativa de Esforço}

A estimativa de esforço obtida a partir da estimativa de tamanho em PCU é calculada considerando outro fator chamado de Produtividade (PROD). Este fator significa a quantidade de horas necessárias para produzir 1 PCU. Desta forma, a estimativa de esforço é obtida com a seguinte fórmula: Esforço = PCU * PROD.

Essa estimativa consiste no esforço das atividades de engenharia para desenvolvimento do software. Para se obter a estimativa de esforço total do projeto, incluindo as atividades de engenharia e gestão, mais dois fatores são considerados: o risco (fator de incerteza do projeto) e a gestão (fator para cálculo do esforço de planejamento e acompanhamento do desenvolvimento do sistema). Dessa forma a estimativa total de esforço é dada pela seguinte fórmula: Esforço $=$ PCU * PROD * GESTÃO * RISCO. 


\section{$4 \quad$ Estudo de Caso}

Uma idéia de evoluir a estimativa baseada na técnica PCU surgiu para considerar diferentes produtividades para diferentes categorias de casos de uso nos projetos de software na empresa E-NOVAR. Essa idéia se baseou na hipótese que casos de uso podem ter complexidades semelhantes $\mathrm{e}$ terem diferentes esforços para desenvolvimento, ou seja, diferentes fatores de produtividade.

Esse estudo de caso analisou três projetos: Sistema de gerenciamento de eventos de uma faculdade (Projeto A), Sistema de gerenciamento financeiro (Projeto B) e Sistema de controle de comissionamento de colaboradores (Projeto C). Os dois primeiros foram usados como estudo e base histórica para a identificação da necessidade da evolução da estimativa baseada na técnica PCU e o último projeto mostra os resultados obtidos com o uso dessa evolução.

\subsection{Projetos A e B}

Os projetos A e B são projetos web e possuem uma quantidade semelhante de casos de uso. O projeto A possui 23 e o projeto B possui 26 casos de uso e seus tamanhos foram 221 e 205 respectivamente. Para ambos foi considerado o fator de produtividade igual a seis, consideramos risco zero para não influenciar e suas ponderações EF e TCF foram parecidas, não gerando impacto neste estudo.

Foi feita a coleta das horas realizadas de todas as atividades dos dois projetos. Neste momento do trabalho serão mostradas as coletas de esforço de desenvolvimento de alguns casos de uso de diferentes categorias juntamente com suas complexidades estimadas.

Tabela 5 - Amostra de complexidades, esforços e desvios dos projetos A e B

\begin{tabular}{|c|c|c|c|c|c|c|c|c|c|c|c|}
\hline & $\begin{array}{l}\text { Descrição } \\
\text { dos Casos } \\
\text { de Uso }\end{array}$ & $\begin{array}{c}U U C \\
W\end{array}$ & $\begin{array}{c}\text { HORAS } \\
\text { PLANEJADA } \\
\text { S }\end{array}$ & $\begin{array}{c}\text { HORAS } \\
\text { REALIZADA } \\
\text { S }\end{array}$ & $\begin{array}{l}\text { DESVI } \\
\text { O (\%) }\end{array}$ & & $\begin{array}{c}\text { Descrição } \\
\text { dos Casos de } \\
\text { Uso }\end{array}$ & $\begin{array}{c}U U C \\
W\end{array}$ & $\begin{array}{c}\text { HORAS } \\
\text { PLANEJADA } \\
\mathbf{S}\end{array}$ & $\begin{array}{c}\text { HORAS } \\
\text { REALIZADA } \\
\mathbf{S}\end{array}$ & $\begin{array}{l}\text { DESVI } \\
\text { O (\%) }\end{array}$ \\
\hline \multirow{9}{*}{$\begin{array}{l}\frac{1}{0} \\
\text { 늠 } \\
\text { 임 } \\
\frac{x}{\alpha}\end{array}$} & $\begin{array}{l}\text { Manter } \\
\text { Participantes }\end{array}$ & 5 & 20,0131 & 32,25 & $61 \%$ & \multirow{9}{*}{ 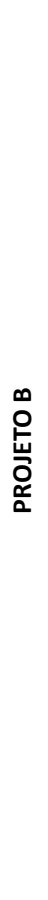 } & $\begin{array}{l}\text { Cadastrar } \\
\text { Convênio }\end{array}$ & 5 & 17,7018 & 31 & $75 \%$ \\
\hline & $\begin{array}{l}\text { Manter } \\
\text { Eventos }\end{array}$ & 10 & 40,0262 & 60,5 & $51 \%$ & & $\begin{array}{l}\text { Cadastrar } \\
\text { Tipo de } \\
\text { Recebimento }\end{array}$ & 10 & 35,4037 & 59,5 & $68 \%$ \\
\hline & $\begin{array}{l}\text { Manter } \\
\text { Trabalhos }\end{array}$ & 15 & 60,0394 & 91 & $52 \%$ & & $\begin{array}{l}\text { Cadastrar } \\
\text { Caixas }\end{array}$ & 15 & 53,1056 & 92 & $73 \%$ \\
\hline & $\begin{array}{l}\text { Inscrever em } \\
\text { Evento }\end{array}$ & 5 & 20,0131 & 25,3 & $26 \%$ & & $\begin{array}{l}\text { Atualizar } \\
\text { Caixas }\end{array}$ & 5 & 17,7018 & 24 & $36 \%$ \\
\hline & $\begin{array}{l}\text { Configurar } \\
\text { Submissão } \\
\text { de Trabalhos }\end{array}$ & 10 & 40,0262 & 49,5 & $24 \%$ & & $\begin{array}{l}\text { Gerar } \\
\text { Negociação }\end{array}$ & 10 & 35,4037 & 51,5 & $45 \%$ \\
\hline & $\begin{array}{l}\text { Configurar } \\
\text { Avaliação }\end{array}$ & 15 & 60,0394 & 76 & $27 \%$ & & $\begin{array}{l}\text { Validar Lote } \\
\text { de } \\
\text { Arrecadação }\end{array}$ & 15 & 53,1056 & 74 & $39 \%$ \\
\hline & $\begin{array}{l}\text { Gerar } \\
\text { Relatório } \\
\text { Geral }\end{array}$ & 5 & 20,0131 & 21 & $5 \%$ & & $\begin{array}{l}\text { Consultar } \\
\text { Importação } \\
\text { de } \\
\text { Arrecadação }\end{array}$ & 5 & 17,7018 & 21 & $19 \%$ \\
\hline & $\begin{array}{l}\text { Gerar } \\
\text { Relatório das } \\
\text { Médias de } \\
\text { Trabalho }\end{array}$ & 10 & 40,0262 & 40,5 & $1 \%$ & & $\begin{array}{l}\text { Consultar } \\
\text { Lançamentos }\end{array}$ & 10 & 35,4037 & 39,5 & $12 \%$ \\
\hline & $\begin{array}{l}\text { Gerar } \\
\text { Relatórios } \\
\text { Painéis }\end{array}$ & 15 & 60,0394 & 60,6 & $1 \%$ & & $\begin{array}{l}\text { Imprimir } \\
\text { Boleto }\end{array}$ & 15 & 53,1056 & 60,5 & $14 \%$ \\
\hline
\end{tabular}




\begin{tabular}{|c|c|c|c|c|c|c|c|c|c|}
\hline $\begin{array}{l}\text { Importar } \\
\text { Notas }\end{array}$ & 5 & 20,0131 & 35 & $75 \%$ & $\begin{array}{l}\text { Importar } \\
\text { Lançamentos }\end{array}$ & 5 & 17,7018 & 34,5 & $95 \%$ \\
\hline $\begin{array}{l}\text { Importar } \\
\text { Trabalhos }\end{array}$ & 10 & 40,0262 & 70,5 & $76 \%$ & $\begin{array}{l}\text { ler } \\
\text { transações }\end{array}$ & 10 & 35,4037 & 71 & $101 \%$ \\
\hline $\begin{array}{l}\text { Ler } \\
\text { configuração } \\
\text { Evento }\end{array}$ & 15 & 60,0394 & 104,5 & $74 \%$ & $\begin{array}{l}\text { Capturar } \\
\text { Pagamentos }\end{array}$ & 15 & 53,1056 & 106 & $100 \%$ \\
\hline
\end{tabular}

A partir das coletas realizadas foi possível observar um desvio entre os esforços planejado e realizado, mostrando uma média de $48 \%$ de desvio. Também foi possível observar um padrão desse desvio entre as categorias de casos de uso, essência desse trabalho.

\subsection{Estimativa de Software Baseada em Produtividade por Categoria de Caso de Uso}

Para ambos os projetos foram calculadas as produtividades reais dividindo cada esforço realizado pelo seu tamanho. Assim, visando ter uma produtividade única por tipo de caso de uso, foi calculada uma média por este tipo em cada projeto, como mostra na tabela a seguir:

Tabela 6 - Produtividades nos projetos A e B por categoria de caso de uso

\begin{tabular}{|c|c|c|c|c|c|}
\hline & CATEGORIA UC & $\begin{array}{l}\text { PRODUTIVIDADE } \\
\text { MÉDIA }\end{array}$ & & CATEGORIA UC & $\begin{array}{l}\text { PRODUTIVIDADE } \\
\text { MÉDIA }\end{array}$ \\
\hline \multirow{4}{*}{ 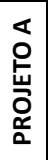 } & CRUD & 6,1889 & \multirow{4}{*}{$\begin{array}{l}\infty \\
o \\
o \\
\frac{0}{0} \\
o \frac{\alpha}{\alpha} \\
\end{array}$} & CRUD & 6,0944 \\
\hline & PADRÃO & 5,0256 & & PADRÃO & 4,9611 \\
\hline & RELATÓRIO & 4,0967 & & RELATÓRIO & 4,0611 \\
\hline & IMPORTAÇÃO/EXPORTAÇÃO & 7,0056 & & IMPORTAÇÃO/EXPORTAÇÃO & 7,0222 \\
\hline
\end{tabular}

Como passo seguinte foi calculada a média das produtividades para aplicação em projetos futuros obtendo-se os seguintes valores:

Tabela 7 - Produtividade médias dos projetos A e B

\begin{tabular}{|l|c|}
\hline \multicolumn{1}{|c|}{ Categoria Caso de Uso } & PROD \\
\hline CRUD & 6,1417 \\
\hline PADRÃO & 4,9933 \\
\hline RELATÓRIO & 4,0789 \\
\hline IMPORTAÇÃO / EXPORTAÇÃO & 7,0139 \\
\hline
\end{tabular}

A evolução da estimativa baseada na técnica PCU preserva todos os cálculos já demonstrados, porém agora considerando produtividades diferentes de acordo com a categoria que o caso de uso pertence. Assim, ao invés de um único valor de produtividade, este trabalho propõe a utilização de mais de um valor de produtividade tantas quantas forem as categorias de casos de uso identificadas.

\subsection{Utilizando a Planilha UCP Evoluída no Projeto C}

A planilha evoluída de PCU foi organizada de modo que as produtividades consideradas na empresa ficassem com os seguintes valores:

Tabela 8 - Produtividade utilizada na organização

\begin{tabular}{|c|c|c|}
\hline CATEGORIA UC & DESCRIÇÃO & PROD \\
\hline CRUD & Produtividade a ser usada por PCU em Caso de Uso CRUD & 6 \\
\hline Padrão & Produtividade Padrão a ser usada por PCU em Casos de Uso & 5 \\
\hline Relatório & Produtividade a ser usada por PCU em Caso de Uso de Relatório & 4 \\
\hline Importação/Exportação & Produtividade a ser usada por PCU em Caso de Uso de Importação/Exportação & 7 \\
\hline
\end{tabular}


O projeto piloto é web, possui 15 casos de uso, seu tamanho é de 123 PCU, possui risco zero e seus fatores técnicos e ambientais estão bem balanceados.

Desta forma, os dados do projeto $\mathrm{C}$ foram coletados da mesma forma que nos projetos $\mathrm{A}$ e $\mathrm{B}$, como mostra a tabela a seguir:

Tabela 9 - Amostra de complexidade e esforços do projeto C

\begin{tabular}{|c|c|c|c|c|c|c|}
\hline & Descrição dos Casos de Uso & Categoria UC & UUCW & $\begin{array}{c}\text { HORAS } \\
\text { PLANEJADAS }\end{array}$ & $\begin{array}{c}\text { HORAS } \\
\text { REALIZADAS }\end{array}$ & $\begin{array}{l}\text { DESVIO } \\
\text { (\%) }\end{array}$ \\
\hline \multirow{11}{*}{ 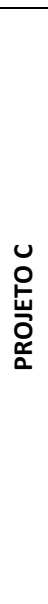 } & $\begin{array}{l}\text { Manter Parâmetro Mecânico } \\
\text { Seminovos }\end{array}$ & \multirow{3}{*}{ CRUD } & 5 & 13,65 & 14 & $3 \%$ \\
\hline & $\begin{array}{l}\text { Manter Parâmetro Vendedor } \\
\text { Seguradora }\end{array}$ & & 10 & 27,31 & 26,5 & $-3 \%$ \\
\hline & $\begin{array}{l}\text { Manter Parâmetro Avaliador } \\
\text { Seminovos }\end{array}$ & & 15 & 40,96 & 38,6 & $-6 \%$ \\
\hline & Manter Seguradora & \multirow{3}{*}{ PADRÃO } & 5 & 16,39 & 17 & $4 \%$ \\
\hline & Manter Seguro & & 10 & 32,77 & 31 & $-5 \%$ \\
\hline & Manter Corretora & & 15 & 49,15 & 50,5 & $3 \%$ \\
\hline & $\begin{array}{l}\text { Relatório comissão de mecânico } \\
\text { Seminovos }\end{array}$ & \multirow{3}{*}{ RELATÓRIO } & 5 & 10,92 & 11,2 & $3 \%$ \\
\hline & $\begin{array}{l}\text { Relatório comissão vendedor } \\
\text { seguradora }\end{array}$ & & 10 & 21,85 & 22 & $1 \%$ \\
\hline & $\begin{array}{l}\text { Relatório comissão avaliador } \\
\text { Seminovos }\end{array}$ & & 15 & 32,77 & 31,5 & $-4 \%$ \\
\hline & Inclusos em NF & \multirow{2}{*}{$\begin{array}{l}\text { IMPORTAÇÃO/ } \\
\text { EXPORTAÇÃO }\end{array}$} & 5 & 19,11 & 20 & $5 \%$ \\
\hline & Exportar Arquivo Folha & & 10 & 38,23 & 37,5 & $-2 \%$ \\
\hline
\end{tabular}

Com esses resultados demonstrados é provado que a utilização das diferentes produtividades por categorias de caso de uso agrega um melhor desempenho.

Para melhor demonstrar o sucesso conseguido com o uso desta evolução da técnica, foi simulado no projeto $\mathrm{C}$ o uso da produtividade igual a seis para todos os tipos de categorias de caso de uso. O resultado da média do desvio desta simulação é mostrado na tabela a seguir juntamente com a média do desvio conseguido com o uso da técnica evoluída proposta:

Tabela 10 - Desvios médios obtidos no projeto $\mathrm{C}$ com o uso de produtividade fixa e com o uso de produtividade diferente por categoria

\begin{tabular}{|l|c|c|c|c|c|c|c|c|}
\hline \multirow{2}{*}{} & \multicolumn{2}{|c|}{ CRUD } & \multicolumn{2}{c|}{ PADRÃo } & \multicolumn{2}{c|}{ RELATÓRIO } & \multicolumn{2}{c|}{$\begin{array}{c}\text { IMPORTAÇÃO / } \\
\text { EXPORTAÇÃO }\end{array}$} \\
\cline { 2 - 9 } & TP & TUC & TP & TUC & TP & TUC & TP & TUC \\
\hline $\begin{array}{l}\text { Desvio Médio } \\
\text { (\%) }\end{array}$ & $-18 \%$ & $-2 \%$ & $0 \%$ & $0 \%$ & $-33 \%$ & $0 \%$ & $18 \%$ & $1 \%$ \\
\hline
\end{tabular}

TP = Técnica padrão UCP / TUC = Técnica UCP com produtividade por categoria de caso de uso

Assim é possível observar que a estimativa do projeto $\mathrm{C}$ foi mais real quando se utilizou produtividades diferentes por categoria de caso de uso, pois suas horas realizadas ficaram próximas de suas horas planejadas, ou seja, o projeto evoluiu ao ponto de ter seu desvio médio de esforço se aproximando à zero por cento, valor ideal para os projetos.

\section{Conclusões}

Este trabalho apresentou a evolução da estimativa baseada na técnica PCU para considerar diferentes fatores de produtividade para diferentes categorias de caso de uso. 
Nos projetos A e B, onde foi usada a técnica padrão de estimativa PCU, foi observado desvio de esforço significativo entre o que havia sido planejado e o que foi realizado.

No projeto $\mathrm{C}$ foi possível aplicar a evolução da técnica de estimativa usando diferentes fatores de produtividade para diferentes categorias de caso de uso, obtendo melhoria nas estimativas, proporcionando mais segurança na execução do projeto e comprometimento das equipes envolvidas.

Como principais trabalhos futuros foram identificados:

- Elaboração de uma base histórica com o uso da evolução da técnica para ter mais dados visando melhores calibrações nas produtividades dos casos de uso.

- Elaboração de novas categorias de casos de uso, visando uma melhor estimativa para a empresa.

- Definição de diferentes produtividades considerando as tecnologias e o processo usado no projeto.

\section{Referências}

HEIMBERG, V., GRAHL E.A., 2005, Estudo de Caso de Aplicação da Métrica de pontos de Caso de Uso numa Empresa de Software, XIV Seminário de Computação - SEMINCO, Outubro

YIN, R.K., 2001, Estudo de caso: planejamento e métodos, $2^{\mathrm{a}}$.edição, Bookman, Porto Alegre, Brasil.

DeMARCO, T. Controle de Projetos de Software. 9.ed. Rio de Janeiro: Editora Campus, 1991

VAZQUEZ, C. E., SIMÕES, G. S., ALBERT, R. M., Análise de Pontos de Função Medição, Estimativas e Gerenciamento de Projetos de Software. $3^{\text {a }}$. edição. São Paulo: Editora Érica, 2005

MONTEIRO, T.C., 2005, Pontos de Caso de Uso Técnico (TUCP): Uma Extensão da UCP. Dissertação de Mestrado, Universidade de Fortaleza.

PEREIRA R., TAIT T.F.C., CASTRO C.Y.H., TRINDADE D.F.G., SILVA J.V., 2007, Estimativas de Software: O Estudo de uma Aplicação Prática Utilizando a Técnica de Use Case Points, Departamento de Informática, Universidade Estadual de Maringá (UEM), Maringá, Brasil.

RIBU K, 2001, Estimating Object-Oriented Software Projects with Use Cases. 